\title{
Sopra una classe di funzionali che approssimano l'area di una superficie.
}

\author{
Nota di Calogero Vinti (a Palermo) (1)
}

A Giovanni. Sansone nel suo $70^{\text {mo }}$ compleanno.

Sunto. - Si introducono per via assiomatica, per le superficie continue $z=f(x, y)$, dei fun. zionali che convergono in area. Si fa vedere poi che non soltanto le medie integrali del primo ordine già usate dal Radò e $i$ polinomi di Stieltjes.Tonelli (e non più «successioni. di essi) rientrano in tale categoria di funzionali, ma anche, tra l'altro, le medie integrali d'ordine $r$, qualunque sia $r \neq 0$.

\section{Introduzione. - L. ToneliL ha caratterizzato $\left({ }^{2}\right)$ le superficie}

$$
z=f(x, y), \quad(x, y) \subset Q\left[a_{0} \leq x \leq b_{0} ; \quad c_{0} \leq y \leq d_{0}\right]
$$

continue ed ad area finita secondo LEBESGUE, mediante la nozione di funzione di due variabili a variazione limitata e, poi, con la nozione di funzione di due variabili assolutamente continua, ha caratterizzato $\left({ }^{3}\right)$ le superficie (1) ad area finita per le quali tale area è espressa mediante un integrale classico.

Successivamente per le superficie (1), continue ed ad area finita, ha mostrato $\left({ }^{4}\right)$, facendo ricorso ad una opportuna generalizzazione dei polinomi di StreltJes $\pi_{n}(x, y)$, relativi alla funzione $f(x, y)$, che l' area $\mathfrak{A}\left[z=\pi_{n}(x, y), R\right]$ della parte di superficie $z=\pi_{n}(x, y)$ che corrisponde al rettangolo $R$ (essendo $R$ un qualsivoglia rettangolo immerso in $Q$ ) tende, per $n \rightarrow \infty$, all'area $\mathfrak{A}[z=$ $=f(x, y), R]$ della parte corrispondente della superficie (1).

Indipendentemente e contemporaneamente, T. RADò, facendo ricorso alle medie integrali del primo ordine:

$$
f_{n}(x, y)=n^{2} \int_{0}^{\frac{1}{n}} \int_{0}^{\frac{1}{n}} f(x+\eta, y+t) d \eta d t
$$

(1) Lavoro eseguito nel Seminario di Analisi Matematica della Università di Palermo. Ringrazio il Prof. E. Baiada per i consigli che mi ha dato.

() L. Tonelli, Sulla quadratura delle superficie (Nota $1^{a}$ ), "Atti Accad. Naz. Lincei Rend. Cl. Sci. Fis. Mat. Nat. (6) 3, $357-362$ (1926).

(') L. Tonelli, Sulla quadratura delle superficie (Nota 2a), a Atti Accad. Naz. Lincei Rend. Cl. Sci. Fis. Mat. Nat. » (6) 3, 633-638 (1926).

(') L. ToNeLta, Su un polinomio di approssimazione e l'area di una superficie, a Atti Accad. Naz. Lincei Rend. Cl. Sei. Fis. Mat. Nat. (6) 5, 313-318 (1927). 
ha mostrato (s), per le superficie (1) continue ed ad area finita, che l'area $\mathfrak{A}\left[z=f_{n}(x, y), R\right]$ tende, al divergere di $n$, all' area $\mathfrak{A}[z=f(x, y), R]$.

In questo lavoro daremo per via assiomatica, nei numeri $2,3,4$, un teorema di approssimazione in area per le superficie continue ed ad area finita, introdncendo dei funzionali che, quando godono di opportune proprietà, ci assicurano la convergenza in area, e, nei numeri 5,6 , faremo vedere che sia i polinomi di StIELdJES-Toneldi, che le medie integrali d'ordine $r$ :

$$
\left\{\frac{1}{h \bar{k}} \int_{0}^{h} \int_{0}^{k} f^{r}(x+\eta, y+t) d \eta d t\right\}^{\frac{1}{r}}
$$

$r$ reale diverso da zero, soddisfano alle proprietà di tali funzionali.

La nostra proposizione assiomatica, mentre da una parte inquadra i due teoremi di approssimazione di ToNELLI-RADò in un unico procedimento, dall'altra generalizza notevolmente il risultato Tonecur-Radò ( ${ }^{\circ}$ ) appunto perchè vale per qualsivoglia funzione di approssimazione che sia un funzionale da noi caratterizzato.

Osserviamo che una generalizzazione diversa del teorema di approssima. zione di ToNELLI-Radò, è stata fatta per le funzioni $f(x, y)$ quasi continne in $Q$.

Poichè per le funzioni quasi continue l'area secondo LeBesgue è in generale privo di significato, L. Cesari (7) ha dato una definizione $\Phi_{C}(f)$ di area generalizzata finita, e, con la nozione di funzione generalmente a variazione limitata, ha caratterizzato le superficie $z=f(x, y)$ ad area generalizzata finita.

Successivamente C. GoFfman, introducendo in modo diverso dal Ceisari l' area $\Phi_{G}(f)$ generalizzata finita $\left({ }^{8}\right)$ (e lo stesso Gofrman $\left({ }^{\circ}\right)$ ha fatto vedere essere equivalente a quella introdotta dal CESARI), ha dato un teorema di

(5) T. Radó, Sur le calcul de l'aire des surfaces courbes, "Eundamenta Mathematicae " vol. 10 (1927), pp. 197.210.

T. RAdó, Length and Area, "American Math. Society Colloquium Publications *, vol. XXX, 1948, pp. 515.516.

(6) Va osservato che la nostra proposizione, anche nel caso particolare delle medie inte. grali del primo ordine, generalizza il risultato di Radó perchè lo medie integrali del primo ordine prese in esame dal RADó sono con $h=k=\frac{1}{n}$.

(7) L. Cesari, Sulle funzioni a variazione limitata, a Annali della Scuola Normale Superiore di Pisa», serie II, vol. V, 1936, pp. 299313.

L. Cesari, Surface Area, Princeton, New Jersry, * Princeton University Press , 19n̆6, pp. 23.24 .

(8) C. Goffman, Lower semi-continuity and area functionals, $I$. The non parametric case, "Rend. Circolo Mat." Palermo, serie 2, vol. 5 (1953), pp. 203-235.

$\left({ }^{\circ}\right)$ C. Goffman, Cfr. nota citata in $\left({ }^{(}\right)$ 
C. VINTI: Sopra una classe di funzionali che approssimano l'area, ecc. 239

approssimazione mostrando $\left({ }^{10}\right)$ che l'area $\mathfrak{A}\left[z=f_{n}(x, y), R\right)$ tende, per $n \rightarrow \infty$, all'area $\Phi_{G}(f) \equiv \Phi_{c}(f)$. La proposizione assiomatica stabilita da noi potrebbe estendersi, senza eccessiva difficolta, alle superficie $z=f(x, y)$, con $f(x, y)$ quasi continue e con area generalizzata finita secondo CESARI-GoFfMAN.

Osserviamo infine, con riferimento ad una comunicazione di G. Trroomi $\left({ }^{11}\right)$ al Congresso di Edinburgh, che la proposizione assiomatica stabilita in questo lavoro porta una economia di pensiero, poichè, come prima è stato detto, oltre a generalizzare notevolmente i risultati di ToNELLI e RADó, unifica i due diversi procedimenti.

2. Denotiamo con $Q$ il rettangolo $\left.\mid 0 \leq x \leq b_{0}<\frac{1}{2} ; \quad 0 \leq y \leq d_{0}<\frac{1}{2}\right]$, con $R[a \leq x \leq b ; c \leq y \leq d]$ un rettangolo completamente interno a $Q$, con $[L Q]$ la classe delle funzioni di due variabili integrabili secondo LEBESGUE in $Q$, con [CVLTQ] la classe delle funzioni di due variabili, continue e a variazione limitata secondo Tonelur in $Q$, con $[A C T R]$ la classe delle funzioni di due variabili assolutamente continue secondo ToNELLI in $R\left({ }^{13}\right)$.

Siano $T_{(x, y)}^{(n, t)}, \mathfrak{N K}_{(x, y)}^{(h, k)}, \overline{\mathfrak{T R}}_{(x, y)}^{(\sigma, h, k)}$ tre funzionali che godono delle seguenti proprietà :

$\left.1^{\circ}\right) T_{(x, y)}^{(n, t)}$ risulti definito per ogni funzione appartenente a $[L Q]$ con la seguente legge: $T_{(x, y)}^{(-,, t)} f(x, y)=f(x+\eta, y+t)$.

$\left.2^{\circ}\right)$ esiste un $\tau>0$ tale che $\mathfrak{T R}_{(x, y)}^{(h, k)}$ risulti definito in [CVLTQ] con valori in $[A C T R]$, per ogni $h$ e $k, 0<h \leq \tau, 0<k \leq \tau$, e per $(x, y) \subset R$.

$\left.3^{0}\right) \lim _{h \rightarrow 0} \mathscr{N}_{(x, y)}^{(h, k)} f(x, y)=f(x, y)$ uniformemente in $R$ e qualunque sia $f(x, y)$ di $[\overrightarrow{C V L T Q}]$.

$4^{\circ}$ ) fissati ad arbitrio $\sigma, h, k$, positivi, esistono due numeri positivi $\gamma(\sigma, h), \gamma^{\prime}(\sigma, k)$ infinitesimi con $\sigma, h, k$, tale the il funzionale $\overline{\mathfrak{T}}_{(x, y)}^{(\sigma, h, k)}$ applicato alla funzione $T_{(x, y)}^{(n, t)} f(x, y)$ della classe $[L Q]$, appartenga alla classe $[L Q]$, al variare di $|\eta| \Theta|t|$ rispettivamente in $(0, \gamma),\left(0, \gamma^{\prime}\right)$.

$5^{\circ)} \mathfrak{O T}_{(x, y)}^{(k, h, k)} T_{(x, y)}^{(n, t)}$ risuiti monotono in $[L Q]$, cioè :

a) $\overline{\mathfrak{9}} \overline{\mathcal{C}}_{(x, y)}^{(\sigma, h, k)} T_{(x, y)}^{(\eta, t)} f(x, y) \leq \overline{\mathfrak{N}}_{(x, y)}^{(\sigma, h, k)} T_{(x, y)}^{(n, t)} g(x, y)$ quando è : $f(x, y) \leq g(x, y)$ per $(x, y) \subset Q$;

(10) C. Gorman, Convergence in area of integral means, "Ameriean Journal of Math. ", vol. LXX VII, 3, 1955, pp. 563-574.

(ii) G. TrIcon, Quo vadimus? * Bollettino Unione Mat. Italiana , serie III, Anno XIII, 1958, N. 4 , pp. 583.585.

(12) Ogni funzione $f(x, y)$ definita in $Q$ la definiamo in tutto il piano ponendo per ogni $y$ di $\left(c_{0}, d_{0}\right) f(x, y)=f\left(a_{0}, y\right)$ se $x<a_{0}$, e $f(x, y)=f\left(b_{0}, y\right)$ se $x>b_{0} ;$ e ponendo poi $f(x, y)=0$ per ogni $y<c_{0}$, e per ogni $y>a_{0}$. 
b) $\overline{\mathfrak{T K}}_{(\boldsymbol{x}, y)}^{(\sigma, h, k)} T_{(x, y)}^{(r, t)} f_{1}(x, y) \leq f_{2}(x, y)$ quando è:

c) $T_{(x, y)}^{(\eta, t)} f_{1}(x, y) \leq f_{2}(x, y)$ per $(x, y) \subset Q$, e per $|\eta| \leq \gamma,|t| \leq \gamma^{\prime}$.

L' nguaglianza in b) abbia lnogo quando ha luogo l' uguaglianza in $c$ ), cioè:

d) $\overline{\mathfrak{T C}}_{(x, y)}^{(a, h, k)} T_{(x, y)}^{(\eta, t)} f(x, y)=f(x, y)$ se eे $f(x, y)$ costante in $Q$.

$\left.6^{\circ}\right)$ Per il funzionale $\overline{\mathfrak{K}}_{(x, y)}^{(a, h, k)}$ valga la seguente disuguaglianza (MrNKowsKI):

$$
\begin{aligned}
& \left\{\left(\overline{\mathscr{T K}}_{(x, y)}^{(\sigma, h, k)} T_{(x, y)}^{(\eta, t)} f_{1}\right)^{2}+\left(\overline{\mathscr{N}}_{(x, y)}^{(\sigma, h, k)} T_{(x, y)}^{(\eta, t)} f_{2}\right)^{2}+\ldots+\left(\overline{\mathscr{O K}}_{(x, y)}^{(\sigma, h, k)} T_{(x, y)}^{(n, t)} f_{s}\right)^{2}\right\}^{\frac{1}{2}} \leq \\
& \leq \overline{\mathfrak{M}}_{(x, y)}^{\left(\sigma, h_{2}, k\right)} T_{(x, y)}^{(n, y)}\left\{f_{1}^{2}+f_{2}^{2}+\ldots+f_{s}^{2}\right\}^{\frac{1}{*}} \text {. }
\end{aligned}
$$

7 이 $\overline{\mathscr{N}}_{(x, y)}^{(\sigma, h, k)} T_{(x, y)}^{(n, t)}$ risulti sub-additivo in $[L Q]$, ciò̀ :

$$
\begin{aligned}
& \sum_{i=1}^{s} \overline{\mathfrak{T}}_{(x, y)}^{(\sigma, h, k)} T_{(x, y)}^{(\eta, t)} f_{i}(x, y) \leq \overline{\mathfrak{M}}_{(x, y)}^{(\sigma, h, k)} T_{(x, y)}^{(n, t)} \sum_{i=1}^{s} f_{i}(x, y) ; \\
& \int_{a_{i}}^{a_{i+1}} \overline{\mathscr{N}}_{(x, y)}^{(\sigma, h) k)} T_{(x, y)}^{(n, t)} f(x, y) d x \leq\left\{\overline{\mathscr{M}}_{(x, y)}^{(\sigma, h, k)} T_{(x, y)}^{(n, t)} \int_{a_{i}+x}^{a_{i+1}+x} f(u, y) d u\right\}_{x=0} ; \\
& \int_{c_{i}}^{c_{j+1}} \overline{\mathscr{N}}_{(x, y)}^{(\sigma, h, k)} T_{(x, y)}^{(n, i)} f(x, y) d y \leq\left\{\overline{\mathscr{N}}_{(x, y)}^{(\sigma, h, k)} T_{(x, y)}^{(n, t)} \int_{c_{j}+y}^{c_{j+1}+y} f(x, v) d v\right\}_{y=0}
\end{aligned}
$$

Denotiamo con $R^{*}$ il rettangolo $\left[0 \leq x \leq b+b_{0}+\tau^{*} ; 0 \leq y \leq d+d_{0}+\tau^{*}\right]$ $\left(\tau^{*}>0\right.$ e tale che risulti: $\left.b+b_{0}+\tau^{*}<1, d+d_{0}+\tau^{*}<1\right)$, con $R^{(1)}$ il rettangolo $[a-(b-a) \leq x \leq b+(b-a) ; c-(d-c) \leq y \leq d+(d-c)]$, ottenuto dal rettangolo $R$ mediante simmetrie rispetto ai suoi lati, con $R^{(2)}$ il rettangolo ottenuto da $R^{(1)}$ mediante simmetrie così come $R^{(1)}$ 's è ottenuto da $R$, e cosl proseguendo denotiamo con $R^{(n)}$ il primo rettangolo, ottenuto con tale procedimento, che contenga il rettangolo $R^{*}$. Se $f(x, y)$ è una funzione definita in $R$, definiamo in $R^{(1)}$ la funzione $f_{1}(x, y)$ con la seguente legge: $f_{1}(x, y)=$ $=f(x, y)$ per $(x, y) \subset R$; nei rettangoli $[b \leq x \leq b+b-a, c \leq y \leq d]$, $[a-(b-a) \leq x \leq a, c \leq y \leq d]$, definiamo $f_{1}(x, y)$ rispettivamente mediante simmetrie rispetto ai lati $x=b, x=a$; nei rettangoli $[a-(b-a) \leq x \leq b+(b-a)$, $d \leq y \leq d+(d-c)],[a-(b-a) \leq x \leq b+(b-a), c-(d-c) \leq y \leq c]$ definiamo $f_{1}(x, y)$ rispettivamente mediante simmetrie rispetto ai lati $y=d, y=c$. In $R^{(2)}$ definiamo la funzione $f_{2}(x, y)$ mediante la $f_{1}(x, y)$ così come s' è definita la $f_{1}(x, y)$ mediante la $f(x, y)$, e proseguendo in tal modo denotiamo con $f_{n}(x, y)$ la funzione definita in $R^{(n)}$ mediante la $f_{n-1}(x, y)$. Definiamo infine in $R^{*}$ la funzione $f^{*}(x, y)$ ponendo $f^{*}(x, y)=f_{n}(x, y)$. 
C. VinTI: Sopra una classe di funzionali che approssimano l'area, ecc. 241

In $R^{*}$ la $f^{*}(x, y)$ è $L, C V L T, A C T$, secondo che in $R$ la $f(x, y)$ risulta rispettivamente $L, C V L T, A C T\left({ }^{13}\right)$.

Nel numero 4. dimostreremo il seguente teorema:

Detta $f(x, y)$ una funzione della classe [OVLTQ], se per ogni $\varepsilon>0$ esiste un $\delta(\varepsilon)>0(\delta<\varepsilon)$ tale che per $\sigma<\delta, h<\delta, k<\delta$, si abbia:

$$
\begin{gathered}
V_{x}\left[\mathscr{N}_{(x, y)}^{(k, k)} f^{*} ; a_{i}, a_{i+1}\right] \leq \varphi_{h, k}\left(y, \sigma ; a_{i}, a_{i+1}\right)+ \\
+(1+\varepsilon)\left\{\overline{\mathscr{K}}_{(x, y)}^{(\sigma, h, k)} T_{(x, y)}^{(\eta, t)} V_{u}\left[f^{*}(u, y) ; a_{i}+x, a_{i+1}+x\right]\right\}_{x=0}, \\
V_{y}\left[\mathscr{N T}_{(x, y)}^{(h, k)} f^{*} ; c_{j}, c_{j+1}\right] \leq \bar{\varphi}_{h, k}\left(x, \sigma ; c_{j}, c_{j+1}+\right. \\
+(1+\varepsilon)\left\{\overline{\mathscr{N}}_{(x, y)}^{(\sigma, h, k\rangle} T_{(x, y)}^{(n, t)} V_{v}\left[f^{*}(x, v) ; c_{j}+y, c_{j+1}+y\right] l_{y=0},\right.
\end{gathered}
$$

dove $\left(a_{i}, a_{i+1}\right), i=1,2, \ldots, m,\left(c_{j}, c_{j+1}\right), j=1,2, \ldots, m^{\prime}$, sono due suddivisioni arbitrarie rispettivamente di $(a, b),(c, d), \varphi_{h, k}\left(y, \sigma ; a_{i}, a_{i+1}\right), \bar{\varphi}_{h, k}\left(x, \sigma ; c_{j}, c_{j+1}\right)$ due funzioni non negative, rispettivamente sommabili in $c \leq y \leq d, a \leq x \leq b$, e soddisfa.

centi le proprietà $: \sum_{i=1}^{m} \sum_{j=1}^{m^{\prime}} \int_{c_{j}}^{c_{j+1}} \varphi_{h, k}\left(y, \sigma ; a_{i}, a_{i+1}\right) d y \leq \psi_{h, k}(\sigma), \sum_{i=1}^{m} \sum_{j=1}^{m_{j}^{\prime}} \int_{\boldsymbol{a}_{i}}^{a_{i+1}} \varphi_{h, k}\left(x, \sigma ; c_{j} ; c_{j+1}\right) \cdot d x \leq$

$\leq \psi_{h, k}(\sigma)$, con $\psi_{h, k}$ funzione non negativa e tale che $\lim _{h \rightarrow 0} \psi_{h, k}(\sigma)=0$ per ogni $\sigma$, allora risulta :

$$
\lim _{\substack{h \rightarrow 0 \\ k \rightarrow 0}} \mathfrak{A}\left[z=\mathfrak{O K}_{(x, y)}^{(h, k)} f^{*}, R\right]=\mathfrak{A}[z=f(x, y), R]\left({ }^{14}\right) .
$$

3. Premettiamo un Lemma. - Detto $R_{h, k}$ il rettangolo $[a-h \leq x \leq b+h$; $c-k \leq y \leq d+k]$ (con $h, k$ positivi e minori del più piccolo tra $i$ numeri: $a, c)$, se $f(x, y)$ appartiene alla classe $[C V L T Q], l$ area $\mathfrak{A}\left[z=f^{*}, R_{h, k}\right]$ tende ad $\mathfrak{G}[z=f, R]$ al tendere di $h$ e $k$ a zero.

Osserviamo intanto che se $R^{(h, k)}$ è il rettangolo $[a+h \leq x \leq b-h$; $c+k \leq y \leq d-k]$ l'area $\mathfrak{a}\left[z=f, R^{(h, k)}\right]$ tende ad $\mathfrak{Q}[z=f, R]$. Infatti è : $\mathfrak{A}\left[z=f, R^{(h, k]}\right] \leq \mathfrak{A}[z=f, R]$, ed in virtù della semicontinuità inferiore di $\mathfrak{A}$, che deriva direttamente dalla definizione di area secondo LeBESGUE, risulta: $\lim _{\substack{h \rightarrow 0 \\ k \rightarrow 0}} \mathfrak{A}\left[z=f, R^{(h, k)}\right] \geq \mathfrak{A}[z=f, R]$, la quale associata alla precedente fornisce l'asserto.

Posto: $\quad R_{1} \equiv[b-h \leq x \leq b ; c+k \leq y \leq d-k]$, $R_{2} \equiv[a+h \leq x \leq b-h ; d-k \leq y \leq d]$, $R_{3} \equiv[a \leq x \leq a+h ; c+k \leq y \leq d-k]$, $R_{4} \equiv[a+h \leq x \leq b-h ; c \leq y \leq c+k]$,

(13) La $f^{*}(x, y)$ la definiamo fuori del suo campo di definizione $R^{*}$, cosi come s' ̀̀ fatto per la $f(x, y)$; efr. nota citata in (12).

(ii) Col simbolo $\mathfrak{Q}[z=f(x, y), R]$ denotiamo l'area secondo LEBESGue della superficio $z=f(x, y)$, per $(x, y) \subset R$. 
in corrispondenza ad un $\varepsilon>0$ si può determinare un $\bar{h}>0$ ed un $\bar{k}>0$, tale che se è $0<h \leq \bar{h}, \quad 0<k \leq \bar{k}$, risulti : $\mathfrak{A}\left[z=f, R_{i}\right]<\frac{\varepsilon}{8}$, per $i=1,2,3,4$.

Sia $\bar{R}_{1}\left(\bar{R}_{3}\right)$ il rettangolo simmetrico di $R_{1}\left(R_{3}\right)$ rispetto alla retta $x=b$ $(x=a)$, ed $\bar{R}_{2}\left(\bar{R}_{4}\right)$ il rettangolo simmetrico di $R_{2}\left(R_{4}\right)$ rispetto alla retta $y=d$ $(y=c)$; per la stessa costruzione di $f^{*}(x, y)$ risulta :

$$
\mathfrak{A}\left[z=f^{*}, \bar{R}_{i}\right]<\frac{\varepsilon}{8} \quad(i=1,2,3,4) .
$$

Siano ora $R_{1}^{*}, R_{2}^{*}, R_{3}^{*}, R_{4}^{*}$, i quattro rettangoli rappresentati dall' insieme $R_{h, k}-\left[R^{(h, k)}+R_{1}+\bar{R}_{1}+R_{2}+\bar{R}_{2}+R_{3}+\bar{R}_{3}+R_{4}+\bar{R}_{4}\right]$ ed osserviamo che essendo l' area di una superficie data da un integrale di WeIERstrass-BurKILL, si può determinare un $h^{\prime}>0, k^{\prime}>0, h^{\prime}<\bar{h}, k^{\prime}<\bar{k}$, tale che per $0<h<h^{\prime}$, $0<k<h^{\prime}$, risulti :

$$
\mathfrak{Q}\left[z=f^{*}, R_{i}^{*}\right]<\frac{\varepsilon}{8} \quad(i=1,2,3,4) .
$$

Dalle (4), (5), segue allora $\mathfrak{A}\left[z=f^{*}, R_{h, k}\right]-\mathfrak{d}[z=f, R]<\varepsilon$ per $0<h<h^{\prime}$, $0<k<k^{\prime}$, e ciò dimostra il lemma.

4. Dimostriamo in questo numero il teorema enunciato al numero 2. Suddividiamo gli intervalli $(a, b),(c, d)$ in parti mediante i punti

$$
a=a_{1}<a_{2}<a_{3}<a_{4}<\ldots<a_{m}=b ; \quad c=c_{1}<c_{2}<c_{3}<\ldots<c_{m}=d,
$$

e poniamo:

$$
\begin{aligned}
& \alpha_{i, j}^{\prime}=\int_{\boldsymbol{a}_{i}}^{\boldsymbol{a}_{i+1}} V_{\nu}\left[\mathscr{N C}_{(x, y)}^{(h, k)} f^{*} ; c_{j}, c_{j+1}\right] d x, \quad \beta_{i, j}^{\prime}=\int_{c_{j}}^{c_{j+1}} V_{x}\left[\mathscr{N}_{(x, y)}^{(h, k)} f^{*} ; a_{i}, a_{i+1}\right] d y, \\
& r_{i, j}=\left(a_{i+1}-a_{i}\right)\left(c_{j+i}-c_{j}\right) \text {, }
\end{aligned}
$$

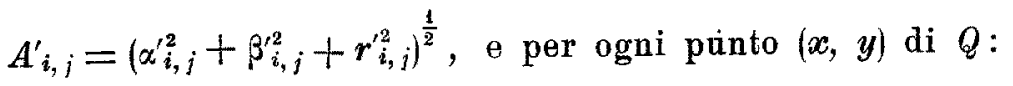

$$
\begin{aligned}
& \alpha_{i, j}(x, y)=\int_{a_{i}+x}^{a_{i+1}+x} V_{v}\left[f^{*}(u, v) ; c_{j}+y, c_{j+1}+y\right] d u, \\
& \beta_{i, j}(x, y)=\int_{c_{j}+y}^{c_{j+1}+y} V_{u}\left[f^{*}(u, v) ; a_{i}+x, a_{i+1}+x\right] d v, \\
& A_{i, j}(x, y)=\left(\alpha_{i, j}^{2}(x, y)+\beta_{i, j}^{2}(x, y)+r_{i, j}^{2}\right)^{\frac{1}{2}} .
\end{aligned}
$$

$\alpha_{i, j}^{\prime}, \beta_{i, j}^{\prime}, \alpha_{i, j}(x, y), \beta_{i, j}(x, y)$ esistono dato che la $f^{*}(x, y)$ è $C V L T$ in $R^{*}$, ed $\mathscr{I T}_{(x, y)}^{(h, k)} f^{*}(x, y)$ əे $A C T$ in $R$. 
C. VINTI: Sopra una classe di funzionali che approssimano l'area, ecc. 243

Integriamo la (2) in $\left(c_{j}, c_{j+1}\right)$, la $(3)$ in $\left(a_{i}, a_{i+1}\right)$, e teniamo conto della proprietà $7^{\circ}$ ) del N. 2 e delle posizioni fatte sopra:

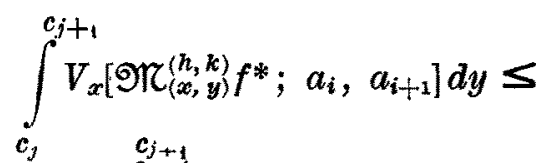

$\leq \int_{c_{j}}^{c_{j+1}} \varphi_{h, k}\left(y, \sigma ; a_{i}, a_{i+1}\right) d y+(1+\varepsilon) \int_{c_{j}}^{c_{j+1}}\left\{\overline{\mathfrak{T}}_{(x, y)}^{(\sigma, h, k)} T_{(x, y)}^{(\eta, t)} V_{u}\left[f^{*}(u, y) ; a_{i}+x, a_{i+1}+x\right]\right\}_{x=0} d y=$ $=\int_{c_{j}}^{c_{j+1}} \varphi_{h, k}\left(y, \sigma ; a_{i}, a_{i+1}\right) d y+(1+\varepsilon)\left\{\int_{c_{j}}^{c_{j+1}} \overline{\mathscr{N}}_{(x, y)}^{(\sigma, h, k)} T_{(x, y)}^{(n, t)} V_{u}\left[f^{*}(u, y) ; a_{i}+x, a_{i+1}+x\right] d y\right\}_{x=0} \leq$ $\left.\leq \int_{c_{j}}^{c_{+j}} \varphi_{h, k}\left(y, \sigma ; a_{i}, a_{i+1}\right) d y+(1+\varepsilon)\left\{\overline{\mathscr{T}}_{(x, y)}^{(\sigma, h, k)} T_{(x, y)}^{(n, t)} \int_{c_{j+1}}^{c_{j+1}+y} V_{u}\left[f^{*}(u, v) ; a_{i}+x, a_{i+1}+x\right] d v\right\}\right\}_{\substack{x=0 \\ y=0}}=$ $=\int_{c_{j}}^{c_{j+1}} \varphi_{h, k}\left(y, \sigma ; a_{i}, a_{i+1}\right) d y+(1+\varepsilon)\left\{\overline{\mathscr{K}}_{(x, y)}^{(\sigma, k, k)} T_{(x, y)}^{(n, t)} \beta_{i, j}(x, y)\right\}_{\substack{x=0 \\ y=0}}$.

$$
\int_{a_{i}}^{a_{i+1}} V_{y}\left[\operatorname{OR}_{(x, y)}^{(h, k)} f^{*} ; c_{j}, c_{j+1}\right] d x \leq
$$

$\leq \int_{a_{i}}^{a_{a_{i+1}}} \bar{\varphi}_{h, k}\left(x, \sigma ; c_{j}, c_{j+1}\right) d x+(1+\varepsilon) \int_{a_{i}}^{a_{i+1}}\left\{\overline{\mathscr{T}}_{(x, y)}^{(\sigma, h, k)} T_{(x, y)}^{(n, t)} \nabla_{v}\left[f^{*}(x, v) ; c_{j}+y, c_{j+1}+y\right]\right\}_{y=0} d x=$ $=\int_{a_{i}}^{a_{i+1}} \bar{\varphi}_{h, k}\left(x, \sigma ; c_{j}, c_{j+1}\right) d x+(1+\varepsilon)\left\{\int_{a_{i}}^{a_{i+t}} \overline{\mathfrak{N}}_{(x, y)}^{(\sigma, h, k)} T_{(x, y)}^{(n, t)} V_{v}\left[f^{*}(x, v) ; c_{j}+y, c_{j+1}+y\right] d x\right\}_{y=0} \leq$ $\leq \int_{a_{i}}^{a_{i+1}} \bar{\varphi}_{h, k}\left(x, \sigma ; c_{j}, c_{j+1}\right) d x+(1+\varepsilon)\left\{\overline{\mathscr{T}}_{(x, y)}^{(\sigma, h, k)} T_{(x, y)}^{(n, t)} \int_{a_{i+1}}^{a_{i+1}+x} V_{v}\left[f^{*}(u, v) ; c_{j}+y, c_{j+1}+y\right] d u\right\}_{\substack{c=0 \\ y=0}}=$ $=\int_{a_{i}}^{a_{i+1}} \bar{\varphi}_{h, k}\left(x, \sigma ; c_{i}, c_{i+1}\right) d x+(1+\varepsilon)\left\{\overline{\mathscr{K}}_{(x, y)}^{(\sigma, k, k)} T_{(x, y)}^{(\eta, t)} \alpha_{i, j}(x, y)\right\}_{\substack{x=0 \\ y=0}}^{a_{i}+x}$.

Dalle $\left(2^{\prime}\right)$ e $\left(3^{\prime}\right)$ segue :

(6)

$$
A_{i, j}^{\prime}=\left(\alpha_{i, j}^{\prime 2}+{\beta_{i, j}^{\prime 2}}^{2}+r_{i, j}^{2}\right)^{\frac{1}{2}}<
$$

$$
\begin{aligned}
& <\left\{\left[\left(\int_{a_{i}}^{a_{i+1}} \varphi_{h, k}\left(x, \sigma ; c_{j}, c_{j+1}\right) d x+(1+\varepsilon) \overline{\mathscr{N}}_{(x, y)}^{(\sigma, h, k)} T_{(x, y)}^{(\mathfrak{n}, t)} \alpha_{i, j}(x, y)\right)^{\varepsilon}+\right.\right. \\
& \left.+\left(\int_{c_{j}}^{c_{j+1}} \varphi_{h, k}\left(y, \sigma ; a_{j}, a_{j+1}\right) d y+(1+\varepsilon) \overline{\mathscr{N}}_{(x, y)}^{(\sigma, h, k)} T_{(x, y)}^{(\eta, t)} \beta_{i, j}(x, y)\right)^{2}+\left.r_{i, j}^{2}\right|_{\substack{x=0 \\
y=0}} ^{\frac{1}{2}}\right\}_{\substack{x=0 \\
y}} .
\end{aligned}
$$


Applicando ora la disuguaglianza di Mrnkowski $\left({ }^{15}\right)$, dalla (6) si perviene alla

$$
\begin{aligned}
& A_{i, j}^{\prime}<\left\{\left[\left(\int_{a_{i}}^{a_{i+1}} \bar{\varphi}_{h, k}\left(x, \sigma ; c_{j}, c_{j+1}\right) d x\right)^{2}+\left(\int_{c_{j}}^{c_{j+1}} \varphi_{h, k}\left(y, \sigma ;-a_{i}, a_{i+1}\right) d y\right)^{2}\right]^{\frac{1}{2}}+\right. \\
& +\mid\left((1+\varepsilon) \overline{\mathfrak{N}}_{(x, y)}^{(\sigma, h, k)} T_{(x, y)}^{(n, t)} \alpha_{i, j}(x, y)\right)^{2}+\left((1+\varepsilon) \overline{\mathscr{N}}_{(x, y)}^{(\sigma, h, k)} T_{(x, y)}^{(n, t)} \beta_{i, j}(x, y)\right)^{2}+ \\
& \left.+r_{i, j}^{2} \mid \frac{1}{\frac{1}{2}}\right\}_{\substack{x=0 \\
y=0}}<\int_{a_{i}}^{a_{i+1}} \bar{\varphi}_{h, k}\left(x, \sigma ; c_{j}, c_{j+1}\right) d x+\int_{c_{j}}^{c_{j+1}} \varphi_{h, k}\left(y, \sigma ; a_{i}, a_{i+1}\right) d y+
\end{aligned}
$$

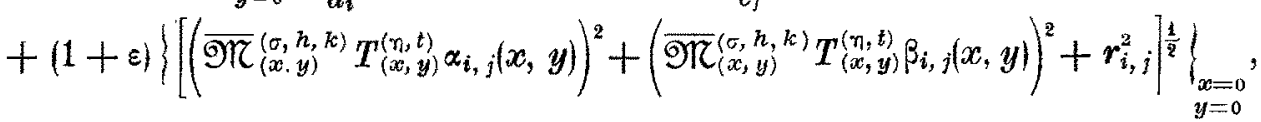

e per le proprietà $5^{\circ}$ ) d) e $6^{\circ}$ ) del N. 2 segue:

$$
\begin{aligned}
& A_{i, j}^{\prime}<\int_{a_{i}}^{a_{i+1}} \varphi_{h, k}\left(x, \sigma ; c_{j}, c_{j+1}\right) d x+\int_{c_{j}}^{c_{j+1}} \varphi_{h, k}\left(y, \sigma ; a_{i}, a_{i+1}\right) d y+ \\
& +(1+\varepsilon)\left\{\overline{\mathscr{K}}_{(x, y)}^{(\sigma, h, k)} T_{(x, y)}^{\left(\eta_{1} t\right)}\left(\alpha_{i, j}^{2}(x, y)+\beta_{i, j}{ }^{2}(x, y)+r_{i, j}^{2}\right)^{\frac{1}{2}}\right\}_{\substack{x=0 \\
y=0}}=
\end{aligned}
$$

$$
\left.=\int_{a_{i}}^{a_{i+1}} \overline{\varphi_{h, k}}\left(x, \sigma ; c_{j}, c_{j+1}\right) d x+\int_{c_{j}}^{c_{j+1}} \varphi_{h, k}\left(y, \sigma ; a_{i}, a_{i+1}\right) d y+(1+\varepsilon)\left\{\overline{\mathscr{K}}_{(x, y)}^{(\sigma, h, k)} T_{(x, y)}^{(\eta, t)} A_{i, j}(x, y)\right\}\right\}_{\substack{x=0 \\ y=0}}
$$

Sommiamo la (7) per $i=1,2,3, \ldots, m ; j=1,2,3, \ldots, m^{\prime}$, teniamo conto della proprieta $7^{\circ}$ ) del N. 2 e delle condizioni a cui soddisfano $\varphi_{h, k}, \varphi_{h, k}$. Otteniamo :

$$
\Sigma A_{i, j}^{\prime}<2 \Psi_{h, k}(\sigma)+(1+\varepsilon)\left\{\overline{\mathscr{K}}_{(x, y)}^{(c, h, k)} T_{(x, y)}^{(n, t)} \Sigma A_{i, j}(x, y)\right\}_{\substack{x=0 \\ y=0}} .
$$

Detto $A^{\prime}$ il confine superiore di tutte le somme $\Sigma A_{i, j}^{\prime}$, ed $A(x, y)$ il confine superiore di tutte le somme $\Sigma A_{i, j}(x, y)$, dalla precedente, in virtù della proprietà $5^{\circ}$ ) a) del N. 2, si deduce :

$$
A^{\prime} \leq 2 \psi_{h, k}(\sigma)+(1+\varepsilon)\left\{\overline{\mathscr{N}}_{(x, y)}^{(\sigma, h, k)} T_{(x, y)}^{(x, t)} A(x, y)\right\}_{\substack{x=0 \\ y=0}} .
$$

Per ogni punto $(x, y)$ di $Q$ denotiamo con $R^{(x, y)}, R_{\varepsilon}^{(x, y)}$ rispettivamente i rettangoli $[a+x, b+x ; c+y, d+y], \quad[a+x-\varepsilon, b+x+\varepsilon ; c+y-\varepsilon$, $d+y+\varepsilon]$ e ricordiamo le seguenti note proprietả stabilite dal ToneLLI $\left({ }^{18}\right)$.

(i5) Cfr. G. H. Hardy, J. E. Litrlewood, G. PolYA, Inequalities, « Cambridge University Press », 1952 pag. 31, formula (2.II.5).

(16) L. Tonelli, Sulla quadratura delle superficie (Nota $2^{a}$ ), a Atti Accad. Naz. Lincei Rend. Cl. Sci. Fis. Mat. Nat." (6), 3, pp. 44ล̌^450 (1926).

L. Tonelun, Ofr. nota citata in ( $\left.{ }^{3}\right)$. 
C. VINTI: Sopra una classe di funzionali che approssimano l'area, ecc. 245

a) $A(x, y) \leq \mathfrak{A}\left[z=f^{*}(u, v), R^{(x, y)}\right] ; A^{\prime} \leq \mathfrak{Q}\left[z=\mathfrak{M K}_{(x, y)}^{(n, k)} f^{*}, R\right]$

b) appartenendo la $\mathfrak{T K}_{(\boldsymbol{x}, y)}^{(h, k)} f^{*}$ alla classe $[A C T R]$, l' area $\mathfrak{E}\left[z=\mathfrak{T K}_{(x, y)}^{(h, k)} f^{*}, R\right]$. è data dall' integrale classico.

c) $\mathfrak{A}\left[z=\mathfrak{N} \mathfrak{c}_{(x, y)}^{(h, k)} f^{*}, R\right]$ non supera $A^{\prime}$

Supponiamo $\varepsilon<a, \varepsilon<\tau^{*}$; i rettangoli $R^{(x+n, y+t)}$ per ogni $(x, y)$ di $Q$ e qualunque siano $|\eta| \leq \varepsilon,|t| \leq \varepsilon$ sono contenuti in $R^{*}$, e poichè $f^{*}(x, y)$, in. $R^{*}$, è CVLT, esistono finite le aree $\mathfrak{d}\left[z=f^{*}(u, v), R^{(x+n) y+t)}\right]$.

Essendo poi $\gamma(\sigma, h)\left[\gamma^{\prime}(\sigma, k)\right]$ infinitesima con $\sigma$ ed $h[k]$ possiamo supporre $\delta(\varepsilon) \operatorname{così~piccolo~che~per~} \sigma<\delta, h<\delta[k<\delta]$, risulti $\gamma \leq \varepsilon\left[\gamma^{\prime} \leq \varepsilon\right]$. Si ha allora:

$$
T_{(x, y)}^{(\eta, t)} A(x, y) \leq T_{(x, y)}^{(\eta, t)} \mathfrak{d}\left[z=f^{*}(u, v), R^{(x, y)}\right] \leq \mathfrak{A}\left[z=f^{*}(u, v), R_{\varepsilon}^{(x, y)}\right]
$$

per $|\eta| \leq \gamma,|t| \leq \gamma^{\prime}$ e qualunque sia il punto $(x, y)$ di $Q$, e per la proprietà $\left.5^{\circ}\right)$ b) risulta :

$$
\overline{\mathfrak{N}}_{(x, y)}^{\left(\sigma, h^{k}\right)} T_{(x, y)}^{(\eta, t)} A(x, y) \leq \mathfrak{Q}\left[z=f^{*}(u, v), R_{\varepsilon}^{(x, y)}\right]
$$

qualunque sia $(x, y) \subset Q$.

In virtù di quest' ultima e delle proprietà $a$ ), b), c), del ToNELLI, dalla (8) segue:

(9) $\mathfrak{\mathbb { X }}\left[z=\mathfrak{N}_{(x, y)}^{(h, k)} f^{*}, R\right] \leq 2 \psi_{h, k}(\sigma)+(1+\varepsilon)\left\{\mathfrak{A}\left[z=f^{*}(u, v), R_{\tilde{\varepsilon}}^{(x, y)}\right]\right\}_{\substack{x=0 \\ y=\theta}}=$

$$
=2 \psi_{h, k}(\sigma)+(1+\varepsilon) \mathfrak{A}\left[z=f^{*}(u, v), R_{\varepsilon}\right],
$$

avendo posto $R_{\varepsilon}=\left\{R_{\varepsilon}^{(x, y)}\right\}_{\substack{x=0 \\ y=0}}$.

Per il lemma dimostrato al N. 3, essendo: $\lim \mathfrak{A}\left[z=f^{*}(u, v), R_{\mathrm{s}}\right]=$ $=\mathfrak{d}[z=f(u, v), R]$, in corrispondenza ad $\eta>0$ arbitrario esiste un $\eta^{*}>0$ (e possiamo supporre $\eta^{*}<\eta$ ) tale che per ogni $0<\varepsilon<\eta^{*}$ risulti: $\mathfrak{a}[z=$ $\left.=f^{*}(u, v), R_{\varepsilon}\right]<\mathfrak{A}[z=f(u, v), R]+\eta$, e quindi dalla (9), supposto $\varepsilon<\eta^{*}$ segue :

$$
\mathfrak{A}\left[z=\mathfrak{N}_{(x, y)}^{(h, k)} f^{*}, R\right]<2 \psi_{h, k}(\sigma)+(1+\varepsilon)\{\mathfrak{A}[z=f(u, v), R]+\eta\} \text {. }
$$

Da quest' ultima tenendo presente ohe $\lim _{\substack{h \rightarrow 0 \\ k \rightarrow 0}} \Psi_{h, k}(\sigma)=0$, si deduce:

$$
\begin{gathered}
\varlimsup_{\substack{h \rightarrow 0 \\
k \rightarrow 0}} \mathfrak{A}\left[z=\mathfrak{K}_{(x, y)}^{(h, k)} f^{*}, R\right] \leq(1+\varepsilon)\{\mathfrak{A}[z=f(u, v), R]+\eta\}< \\
<(1+\eta)\{\mathfrak{A}[z=f(u, v), R]+\eta\}
\end{gathered}
$$

e data l'arbitrarietà di $\eta$ si ha:

$$
\varlimsup_{\substack{h \rightarrow 0 \\ k \rightarrow 0}} \mathfrak{A}\left[z=\operatorname{NK}_{(x, y)}^{(h, k)} f^{*}, R\right] \leq \mathfrak{A}[z=f(u, v), R] .
$$

Quest' ultima dimostra il teorema enunciato al N. 2 osservando che per la proprietà $3^{\circ}$ ) e per la definizione di area secondo LEBEsGue è :

$$
\lim _{\substack{h \rightarrow 0 \\ k \rightarrow 0}} \mathfrak{d}[z=\mathfrak{O K} \underset{(x, y)}{\substack{(h, k) \\ k \rightarrow 0}}, R] \geq \mathfrak{A}\left[z=f^{*}(u, v), R\right]=\mathfrak{d}[z=f(u, v), R]
$$


5. I funzionali $\mathfrak{K}_{(x, y)}^{(h, k)}, \overline{\mathfrak{T}}_{(x, y)}^{(\sigma, h, k)}$ medie integrali. - Sia $f(x, y)$ appartenente alla classe $[C V L T Q]$ e supponiamo che il minimo $N$ di $f(x, y)$ in $Q$ sia positivo (ipotesi questa non restrittiva perchè basta eventualmente traslare la $z=f(x, y)$ lungo l'asse $z)$. Detto $\tau$ il più piccolo tra i numeri $b_{0}-b$, $d_{0}-d$, per ogni $h$ e $k, 0<h<\tau, 0<k<\tau$, e per $(x, y) \subset R$, poniamo

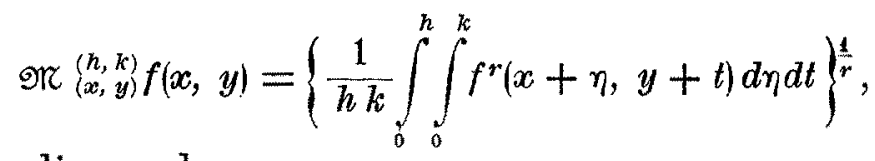

con $r$ reale e diverso da zero.

Se $f(x, y)$ appartiene alla classe $[L Q]$, fissati ad arbitrio $\sigma, h, k$ positivi, posto $\gamma(\sigma, h)=h, \gamma^{\prime}(\sigma, k)=k$, definiamo $\overline{\mathscr{N}}\left(\begin{array}{l}(\sigma, h, k) \\ (x, y)\end{array} T_{(x, y)}^{(\eta, t)} f(x, y)\right.$, per $(x, y)<Q$, ponendo:

$$
\overline{\mathscr{N}}_{(x, y)}^{(\sigma, h, k)} T_{(x, y)}^{(n, t)} f(x, y)=\frac{1}{h k} \int_{0}^{h} \int_{0}^{k} f(x+\eta, y+t) d \eta d t .
$$

La funzione $\mathscr{T K}_{(x, y)}^{(h, k)} f$ soddisfa manifestamente la proprietà $2^{\circ}$ ) del N. 2; per mostrare che è verificata la proprietà $3^{\circ}$ ) del $\mathrm{N}$. 2, osserviamo ohe se $B$ e $C$ sono due numeri positivi, ed $s$ è un numero reale, si hanno le seguenti relazioni $\left({ }^{17}\right)$ :

$$
\begin{array}{ll}
s \cdot B^{s-1}(B-C) \geq B^{s}-C^{s} \geq s \cdot C^{s-1}(B-C) & (\text { per } s \leq 0 \text { od } s \geq 1) \\
s \cdot B^{s-1}(B-C) \leq B^{s}-C^{s} \leq s \cdot C^{s-1}(B-C) & (\text { per } 0 \leq s \leq 1)
\end{array}
$$

e da queste:

$$
\left|B^{s}-C^{s}\right| \leq|s| \cdot D^{s-1} \cdot|B-C|,
$$

ove $D^{s-1}$ denota il più grande tra i numeri $B^{s-1}, C^{s-1}$. È :

$$
\begin{gathered}
\left|\operatorname{IR}_{(x, y)}^{\left(\frac{h}{(x)}\right)} f(x, y)-f(x, y)\right|= \\
=\left|\left\{\frac{1}{h k} \int_{0}^{h} \int_{0}^{k} f^{r}(x+\eta, y+t) d \eta d t\right\}^{\frac{l}{r}}-\left\{\frac{1}{h k} \int_{0}^{h} \int_{0}^{k} f^{r}(x, y) d \eta d t\right\}^{\frac{1}{\eta_{i}}}\right|,
\end{gathered}
$$

ed in virtù della (12) quest' ultima viene maggiorata, qualunque sia $(x, y) \subset R$, come segue

$$
\begin{aligned}
& \mid \mathscr{N}\left(\begin{array}{c}
h, k) \\
(\boldsymbol{x}, y)
\end{array} f(x, y)-f(x, y)\left|\leq \frac{1}{|r|} \bar{M}\right| \frac{1}{h k} \int_{0}^{h} \int_{0}^{k} f^{r}(x+\eta, y+t) d \eta d t-\right. \\
& \left.-\frac{1}{h k} \int_{0}^{h} \int_{0}^{k} f^{r}(x, y) d \eta d t\left|=\frac{1}{|\boldsymbol{r}|} \bar{M}\right| \frac{1}{h k} \int_{0}^{h} \int_{0}^{k} f^{r}(x+\eta, y+t)-f^{\prime}(x, y)\right\} d \eta d t \mid
\end{aligned}
$$

(17) Cfr. G. H. Hardy, J. E. Littlewood, G. Polya, Inequalities (testo citato in 15), pag. 39, formule 2.15.1, 2.15.2. 
C. VINTI: Sopra una classe di funzionali che approssimano l'area, ecc. 247

ove $\bar{M}$ denota un numero che limita superiormente in $R$ le due funzioni:

$$
\left\{\frac{1}{h k} \int_{0}^{h} \int_{0}^{k} f^{r}(x+\eta, y+t) d \eta d t\right\}^{\frac{1}{r}-1}, \quad\left\{\frac{1}{h k} \int_{0}^{h} \int_{0}^{k} f^{r}(x, y) d \eta d t\right\}^{\frac{1}{r}-1},
$$

qualunque siano $h$ e $k$, con $0<h \leq \tau, 0<k \leq \tau$.

$\mathrm{Ma}$ per l'uniforme continuità della $f^{\prime}(x, y)$ in $Q$, in corrispondenza ad $\varepsilon>0$ esiste un $\delta(\varepsilon)>0$ tale che per $0 \leq \eta \leq h<\delta, 0 \leq t \leq k<\delta$, e quaIunque siano $(x, y) \subset R$, si abbia:

$$
\left|f^{\prime}(x+\eta, y+t)-f^{r}(x, y)\right|<\frac{|r| \varepsilon}{\bar{M}}
$$

e quindi dalla (13), per $0<h<\delta, 0<k<\delta$, e per $(x, y) \subset R$, si ha:

$$
\left|\mathfrak{N K}_{(x, y)}^{(h, k)} f(x, y)-f(x, y)\right|<\varepsilon .
$$

La proprietà $3^{\circ}$ ) del N. 2 è dunque dimostrata.

Osserviamo ara che il funzionale $\overline{\mathfrak{N}}_{(x, y)}^{(\sigma, h, k)}$ definito mediante la (11) soddisfa le proprieta $\left.5^{\circ}\right), 6^{\circ}$, $7^{\circ}$ ) del $\mathrm{N}$. 2 perchè, come facilmente, si vede in $[L Q]$ risulta monotono, per esso vale la disuguaglianza di MıNkowski, ed è additivo.

Prima di mostrare che tali funzionali soddisfano le (2), (3) del N. 2 premettiamo un lemma del quale daremo un cenno di dimostrazione.

LeMма. - Se $f(x)$ è continua e a variazione limitata in un intervallo $\left(a_{0}, b_{0}\right)$, preso comunque un intervallo $(a, b)$ interno a $\left(a_{0}, b_{0}\right)$, è :

$$
\int_{a}^{b}\left|\frac{f(x+h)-f(x)}{h}\right| d x \leq \frac{1}{h} \int_{0}^{h} V[f(x) ; a+\eta, b+\eta] d \eta
$$

per ogni $0<h \leq b_{0}-b$, e, dove $V[f(x) ; a+\eta, b+\eta]$ è la variazione totale di $f(x)$ nell' intervallo $(a+\eta, b+\eta)\left({ }^{18}\right)$.

Dividiamo $(a, b)$ in un numero finito d'intervalli consecutivi $\left(a_{i}, b_{i}\right)$, $i=1,2,3, \ldots, n,\left(a_{1}=a, b_{n}=b\right)$, per $0<h \leq b_{0}-b$.

Poniamo $f_{h}(x)=\frac{1}{h} \int_{0}^{h} f(x+\eta) d \eta ;$ risulta allora: $f_{h}^{\prime}(x)=\frac{f(x+h)-f(x)}{h}$, ed essendo. $f_{h}(x)$ assolutamente continua si ha:

$$
V\left[f_{h}(x) ; a, b\right]=\int_{a}^{b}\left|f^{\prime} h(x)\right| d x=\int_{a}^{b}\left|\frac{f(x+h)-f(x)}{h}\right| d x .
$$

(18) Questo lemma è un miglioramento d'un risultato noto, vedi T. RADó, \& Length and Area "(eitato in nota ${ }^{5}$ ), paragrafo III.2.40, relazione (1), pag. 206. La dimostrazione qui data segue le linee di quella riportata dal RADó. 
Ma è per definizione:

e poichè è :

$$
\begin{aligned}
\sum_{i=1}^{n} \mid f_{h}\left(b_{i}\right) & -f_{h}\left(a_{i}\right)\left|=\sum_{i=1}^{n}\right| \frac{1}{h} \int_{0}^{n}\left[f\left(b_{i}+\eta\right)-f\left(a_{i}+\eta\right)\right] d \eta \mid \leq \\
& \leq \frac{1}{h} \int_{0}^{n} \sum_{i=1}^{n}\left|f\left(b_{i}+\eta\right)-f\left(a_{i}+\eta\right)\right| d \eta,
\end{aligned}
$$

$$
\sum_{i=1}^{n}\left|f\left(b_{i}+\eta\right)-f\left(a_{i}+\eta\right)\right| \leq V[f(x) ; a+\eta, b+\eta],
$$

si deduce:

$$
\sum_{i=1}^{n}\left|f_{h}\left(b_{i}\right)-f_{h}\left(a_{i}\right)\right| \leq \frac{1}{h} \int_{0}^{h} V[f(x) ; a+\eta, b+\eta] d \eta .
$$

Osservando che il primo membro di quest'ultima disuguaglianza può essere comunque vicino alla variazione totale della $f_{h}(x)$ su $(a, b)$, risulta:

$$
V\left[f_{h}(x) ; a, b\right]=\int_{a}^{b}\left|\frac{f(x+h)}{h}-f(x)\right| d x \leq \frac{1}{h} \int_{0}^{h} V[f(x) ; a+\eta, b+\eta] d \eta .
$$

Mostriamo ora le (2) e (3) del N. 2. Derivando la (10) rispetto ad $x$, dopo avere sostituito nella $(10) f(x, y)$ con $f^{*}(x, y)$, si ba:

$$
\begin{gathered}
\frac{\partial \mathfrak{N R}_{(x, y)}^{(h, k)} f^{*}}{\partial x}=\frac{1}{r}\left\{\frac{1}{h k} \int_{0}^{h} \int_{0}^{k} f^{r}(x+\eta, y+t) d \eta d t\right\}^{\frac{1-r}{r}} . \\
\cdot \frac{1}{h k} \int_{0}^{k}\left[f^{*}(x+h, y+t)-f^{*}(x, y+t)\right] d t,
\end{gathered}
$$

e quindi:

$$
\begin{gathered}
\left|\frac{\partial \mathscr{N}_{(x, y)}^{(h, k)}}{\partial x}\right| \leq \frac{1}{|r|}\left|\frac{1}{h k} \int_{0}^{k} \int_{0}^{k} f^{*}(x+\eta, y+t) d \eta d t\right|^{\frac{1-r}{r}} \\
\cdot \frac{1}{h k} \int_{0}^{k}\left|f^{*}(x+h, y+t)-f^{*}(x, y+t)\right| d t .
\end{gathered}
$$

Ma per la continuità della $f^{r-1}(x, y)$ in $R^{*}$, ogni qualvolta si fissino $x, y$, $h, k,((x, y) \subset R, 0<h \leq \tau, 0<k \leq \tau)$ esiste un $\mathfrak{y}^{\prime}, 0 \leq \mathfrak{Y}^{\prime} \leq 1$, tale che $f^{r-1}\left(x+h, y+y^{r} \cdot k\right)$ rappresenti il massimo di $f^{r-1}(x+h, y+t)$ al variare di $t$ in $(0, k)$; ed analogamente esiste un $\mathfrak{t}^{\prime \prime}, 0 \leq \mathfrak{q}^{\prime \prime} \leq 1$, tale che $f^{*-1}\left(x, y+\mathfrak{q}^{\prime \prime} \cdot k\right)$ 
C. VintI: Sopra una classe di funsionali che approssimano larea, cce. 249

rappresenti il massimo di $f^{r-1}(x, y+t)$ al variare di $t$ in $(0, k)$. Denotando allora con $G(x, y, h, k)$ il più grande tra i due numeri $f^{*-1}\left(x+h, y+\mathfrak{q}^{\prime} \cdot k\right), f^{r-1}\left(x, y+\vartheta^{\prime \prime} \cdot k\right)$, dalla (12), ogni qualvolta si fissino $x, y, h, k$, si deduce:

$\left|f^{*}(x+h, y+t)-f^{*}(x, y+t)\right| \leq|r| G(x, y, h, k)\left|f^{*}(x+h, y+t)-f^{*}(x, y+t)\right|$, per $0 \leq t \leq k$, e dalla (14) segue:

$$
\begin{gathered}
\left|\frac{\partial \sim_{(x, y)}^{(h, k)}}{\partial x}\right| \leq \frac{1}{|r|}\left\{\frac{1}{h k} \int_{0}^{h} \int_{0}^{k} f^{*}(x+\eta, y+t) d \eta d t\right\}^{\frac{1-r}{r}} . \\
\cdot \frac{1}{h k}|r| G(x, y, h, k) \int_{0}^{k}\left|f^{*}(x+h, y+t)-f^{*}(x, y+t)\right| d t .
\end{gathered}
$$

Intanto per il teorema della media, ogni qualvolta si fissino $x, y, h, k$, esistono un $\vartheta_{1}, 0 \leq \vartheta_{1} \leq 1$, ed un $\vartheta_{2}, 0 \leq \vartheta_{2} \leq 1$, tale che:

$$
\left\{\frac{1}{h k} \int_{0}^{h} \int_{0}^{k} f^{r}(x+\eta, y+t) d \eta d t\right\}^{\frac{1-r}{r}}=\frac{1}{f^{*}\left(x+\vartheta_{1} \cdot h, y+\vartheta_{2} \cdot k\right)},
$$

e la (15) si scrive sotto la forma :

$$
\begin{aligned}
& \left|\frac{\partial \mathfrak{O K}_{(x, y)}^{(h, k)}}{\partial x}\right| \leq \frac{G(x, y, h, k)}{f^{*}\left(x+\vartheta_{1} \cdot h, y+\vartheta_{2} \cdot k\right)} \\
& \cdot \frac{1}{h k} \int_{0}^{k}\left|f^{*}(x+h, y+t)-f^{*}(x, y+t)\right| d t .
\end{aligned}
$$

Per l'uniforme continuità della $f^{r-1}$ in $R^{*}$, in corrispondenza ad $\varepsilon>0$, esiste an $\delta(\varepsilon)>0$ (e possiano supporre $\delta<\tau)$ tale che risulti :

$$
f^{r-1}\left(\xi_{1}, \eta_{1}\right)<f^{*-1}\left(\xi_{2}, \eta_{2}\right)+\varepsilon \bar{N},
$$

per ogni coppia di punti $\left(\xi_{1}, \eta_{1}\right),\left(\xi_{2}, \eta_{2}\right)$ di $R^{*}$, con $\left|\xi_{1}-\xi_{2}\right|<\delta,\left|\eta_{1}-\eta_{2}\right|<\delta$, $\bar{N}>0$ essendo il minimo di $f^{r-1}$ in $R^{*}$; e quindi supposto $0<h<\delta, 0<k<\bar{\theta}$, poichè, qualunque sia $(x, y) \subset R$, per la coppia di punti $\left(x+h, y+\vartheta^{\prime} \cdot k\right)$, $\left(x+\vartheta_{1} \cdot h, y+\vartheta_{2} \cdot h\right)$, si ha:

$$
\begin{gathered}
\left|x+h-\left(x+\vartheta_{1} \cdot h\right)\right|=\left|h\left(1-\vartheta_{1}\right)\right| \leq h<\delta, \\
\left|y+\vartheta^{\prime} \cdot k-\left(y+\vartheta_{2} \cdot k\right)\right|=\left|k\left(\vartheta^{\prime}-\vartheta_{2}\right)\right| \leq k<\delta,
\end{gathered}
$$

e per la coppia di punti $\left(x, y+\vartheta^{\prime \prime} \cdot k\right),\left(x+\vartheta_{1} \cdot h, y+\vartheta_{2} \cdot k\right)$, si ha pure:

$$
\left|x-\left(x+\vartheta_{1} \cdot h\right)\right| \leq h<\delta, \quad\left|y+\vartheta^{\prime \prime} \cdot k-\left(y+\vartheta_{2} \cdot k\right)\right|=\left|k\left(\vartheta^{\prime \prime}-\vartheta_{2}\right)\right| \leq k<\delta,
$$


risulta :

$$
\begin{gathered}
f^{r-1}\left(x+h, y+\vartheta^{\prime}: k\right)<f^{r-1}\left(x+\vartheta_{1} \cdot h, y+\vartheta_{2} \cdot k\right)+\varepsilon \bar{N}, \\
f^{r-1}\left(x, y+\vartheta^{\prime \prime} \cdot k\right)<f^{*-1}\left(x+\vartheta_{1} \cdot h, y+\vartheta_{2} \cdot k\right)+\varepsilon \bar{N},
\end{gathered}
$$

e di conseguenza, tenendo presente la definizione di $G(x, y, h, k)$, per $0<h<\delta$, $0<k<\delta$, e qualunque sia $(x, y) \subset R$, si ha:

$G(x, y, h, k)<f^{r-1}\left(x+\vartheta_{1} \cdot h, y+\vartheta_{2} \cdot k\right)+\varepsilon \bar{N}$, ciò̀ $\frac{G(x, y, h, k)}{f^{r-1}\left(x+\vartheta_{1} \cdot h, y+\vartheta_{2} \cdot k\right)}<1+\varepsilon$.

Dalla (16) supposto $0<h<\delta, 0<k<\delta$, si deduce allora:

$$
\left|\frac{\partial \mathscr{O K}_{(x, y)}^{(h, k)}}{\partial x}\right|<(1+\varepsilon) \frac{1}{h k} \int_{0}^{h}\left|f^{*}(x+h, y+t)-f^{*}(x, y+t)\right| d t .
$$

E con analogo ragionamento:

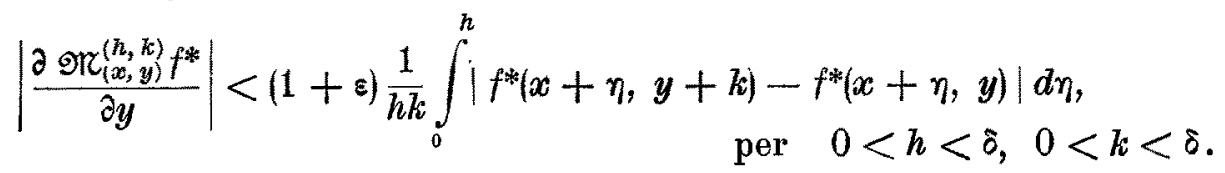

Detti $\left(a_{i}, a_{i+1}\right),\left(c_{j}, c_{j+1}\right)$ rispettivamente due intervalli di $(a, b),(c, d)$, dalle (17) e (18) si ha:

$$
\begin{aligned}
& V_{x}\left[\mathscr{O K}_{(x, y)}^{(h, k)} f^{*} ; a_{i}, a_{i+1}\right]=\int_{a_{i}}^{a_{i+1}}\left|\frac{\partial \mathfrak{N R}_{(x, y)}^{(h, k)} f^{*}}{\partial x}\right| d x< \\
& <(1+\varepsilon) \frac{1}{h k} \int_{a_{i}}^{a_{i+1}} d x \int_{0}^{k}\left|f^{*}(x+h, y+t)-f^{*}(x, y+t)\right| d t \\
& V_{y}\left[\mathscr{Q R}_{(x, y)}^{(h, k)} f^{*} ; c_{j}, c_{j+1}\right]=\int_{e_{j}}^{c_{j+1}}\left|\frac{\partial \operatorname{SR}_{(x, y)}^{(h, k)}}{\partial y}\right| d y< \\
& <(1+\varepsilon) \frac{1}{h k} \int_{c_{j}}^{c_{j+1}} d y \int_{0}^{h}\left|f^{*}(x+\eta, y+k)-f^{*}(x+\eta, y)\right| d \eta,
\end{aligned}
$$

e per un teorema di FubinI-ToNeLLI:

$$
\begin{aligned}
& V_{x}\left[\operatorname{OK}_{(x, y)}^{(h, k)} f^{*} ; a_{i}, a_{i+1}\right]<(1+\varepsilon) \frac{1}{k} \int_{0}^{k} d t \int_{a_{i}}^{a_{i+1}}\left|\frac{f^{*}(x+h, y+t)-f^{*}(x, y+t)}{h}\right| d x \\
& V_{y}\left[\operatorname{RK}_{(x, y)}^{(h, k)} f^{*} ; c_{j}, c_{j+1}\right]<(1+\varepsilon) \frac{1}{h} \int_{0}^{h} d \eta \int_{c_{j}}^{c_{j+1}}\left|\frac{f^{*}(x+\eta, y+k)-f^{*}(x+\eta, y)}{k}\right| d y .
\end{aligned}
$$


C. VINTI: Sopra una classe di funaionali che approssimano l'area, ecc. 251

E infine in virtù del lemma dimostrato in questo numero si ha:

$$
\begin{aligned}
& V_{x}\left[\mathscr{N}_{(h, k)}^{(x, y)} f^{*} ; a_{i}, a_{i+1}\right]<(1+\varepsilon) \\
& \frac{1}{h k} \int_{0}^{h} \int_{0}^{k} V_{x}\left[f^{*}(x, y+t) ; a_{i}+\eta, a_{i+1}+\eta\right] d \eta d t, \\
& V_{y}\left[\mathscr{N R}_{(\boldsymbol{x}, y)}^{(h, k)} f^{*} ; c_{j}, c_{j+1}\right]<(1+\varepsilon) \frac{1}{h k} \int_{0}^{h} \int_{0}^{k} V_{y}\left[f^{*}(x+\eta, y) ; c_{j}+t, c_{j+1}+t\right] d \eta d t,
\end{aligned}
$$

e queste, tenendo presente la definizione del funzionale $\overline{\mathfrak{N}}_{(x, y)}^{(\sigma, h, k)}$ rappresentano le (2) e (3) del N. 2 quando si pone $\varphi_{h, k}=0, \varphi_{h, k}=0, \psi_{h, k}^{\prime}=0$.

6. I funzionali $\mathfrak{N K}_{(x, y)}^{(h, k)}, \overline{\mathfrak{N}}_{(x, y)}^{(\sigma, h, k)}$ polinomi di Stieltjes-Tonelli. - Poniamo $h=k=\frac{1}{n}$, con $u$ intero. Se $f(x, y)$ appartiene alla classe [CVLTQ], qualunque sia l'intero $n$, e per $(x, y) \subset R$, definiamo $\mathscr{N}_{(x, y)}^{\left(\frac{1}{n}, \frac{1}{n}\right)} f$ ponendo:

$$
\begin{gathered}
\mathscr{N}_{(x, y)}^{\left(\frac{1}{n}, \frac{1}{n}\right)} f=\frac{K_{n}^{2}}{4} \iint_{0} f(u, v)\left[1-(u-x)^{2}\right]^{n}\left[1-(v-y)^{2}\right]^{n} d u d v= \\
=\frac{K_{n}^{2}}{4} \int_{-\infty}^{b_{0}-\infty} \int_{-y}^{d_{0}-y} f(x+\eta, y+t)\left(1-\eta^{2}\right)^{n}\left(1-t^{2}\right)^{n} d \eta d t,
\end{gathered}
$$

ove ̀े $K_{n}=\frac{1}{\int_{0}^{1}\left(1-t^{2}\right)^{n} d t}$.

Se $f(x, y)$ appartiene alla classe $[L Q]$, fissati ad arbitrio $\sigma, h, k$ positivi, posto $\gamma(\sigma, h)=\sigma^{*}, \gamma^{\prime}(\sigma, k)=\sigma^{*}$ (essendo $\sigma^{*}$ il primo summultiplo di $\sigma$ minore di 1), definiamo $\overline{\mathfrak{N}}_{(x, y)}^{\left(\sigma, \frac{1}{n}, \frac{1}{n}\right)} T_{(x, y)}^{(n, t)} f$ con la seguente legge:

$$
\begin{aligned}
& \overline{\mathscr{N R}}_{(x, y)}^{\left(\sigma, \frac{1}{n}, \frac{1}{n}\right)} T_{(x, y)}^{(n, t)} f= \\
& =\frac{1}{4\left(\int_{0}^{\sigma^{*}}\left(1-t^{2}\right)^{n} d t\right)^{2}} \int_{-\sigma^{*}}^{\sigma^{*}} \int_{\sigma^{*}}^{\sigma^{*}} f(x+\eta, y+t)\left(1-\eta^{2}\right)^{n}\left(1-t^{2}\right)^{n} d \eta d t .
\end{aligned}
$$

La funzione $\mathscr{O K}_{(x, y)}^{\left(\frac{1}{n}, \frac{1}{n}\right)} f$ soddisfa manifestamente la proprietà $2^{\circ}$ ) del N. 2.

La proprietà $3^{\circ}$ ) del N. 2 è stata dimostrata dal ToneLLI $\left({ }^{19}\right.$ ). Il funzio-

(19) L. TonelLI, Sulla rappresentazione analitica delle funzioni di più variabili reali. "Rendiconti Circolo Matematico di Palermos, Tomo XXIX (1910), I ${ }^{\circ}$ semestre, pp. 2-36.

Ch.-J, de la Valdée Poussin, Conrs d'Analyse Infinitésimale, Tomo, II (1912), pag. 133, Paris, Gauthier-Villars, Editeur. 
252 C. VIntr: Sopra una classe di funsionali che approssimano l'area, ece.

nale $\overline{\mathscr{M}}\left(\sigma, \frac{1}{n}, \frac{1}{n}\right)$ sopra definito soddisfa le proprietà $\left.\left.5^{\circ}\right), 6^{\circ}\right), 7^{\circ}$ ) del N. 2 , perchè, come facilmente si vede, è in $[L Q]$ monotono, per esso vale la disuguaglianza di MINkowskr, ed è additivo.

Se $f(x, y)$ appartiene alla classe [OVLTQ], L. ToneLLI $\left({ }^{20}\right)$ stabilisce le seguenti disuguaglianze:

$$
\begin{aligned}
& V_{x}\left[\mathscr{F R}_{(x, y)}^{\left(\frac{1}{n}, \frac{1}{n}\right)} f^{*} ; a_{i}, a_{i+1}\right] \leq 2 M(n+1)\left(1-\sigma^{2}\right)^{n}\left(a_{i+1}-a_{i}\right)+ \\
& +\frac{K_{n}^{2}}{4} \int_{-1}^{1} \int_{-1}^{1} V_{x}\left[f^{*}(x, y+t) ; a_{i}+\eta, a_{i+1}+\eta\right]\left(1-\eta^{2}\right)^{n}\left(1-t^{2}\right)^{n} d \eta d t, \\
& V_{\nu}\left[\mathscr{N}_{(x, y)}^{\left(\frac{1}{n}, \frac{1}{n}\right)} f^{*} ; c_{j}, c_{j+1}\right] \leq 2 M(n+1)\left(1-\sigma^{2}\right)^{n}\left(c_{j+1}-c_{j}\right)+ \\
& +\frac{K_{n}^{2}}{4} \int_{-1}^{1} \int_{-1}^{1} V_{\nu}\left[f^{*}(x+\eta, y) ; c_{j}+t, c_{j+1}+t\right]\left(1-\eta^{2}\right)^{n}\left(1-t^{2}\right)^{n} d \eta d t
\end{aligned}
$$

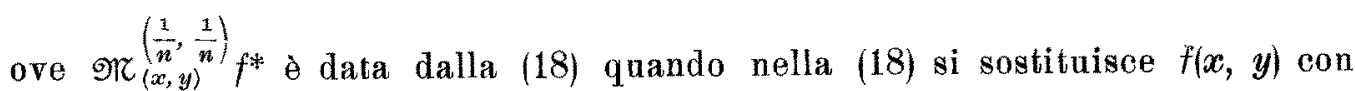
$f^{*}(x, y), Q$ con $R^{*}$, ed essendo: $\left(a_{i}, a_{i+1}\right),\left(c_{j}, c_{j+1}\right)$ due intervalli arbitrari rispettivamente di $(a, b),(c, d) ; M$ il massimo di $f^{*}(x, y)$ in $R^{*}$, e $0<\sigma<1$ un numero arbitrario.

Osserviamo che il secondo addendo del secondo membro della (20) si scrive:

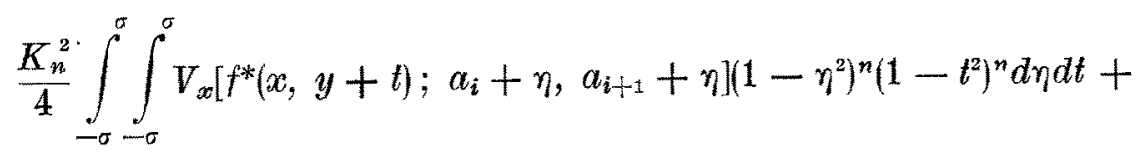

$$
\begin{aligned}
& +\frac{K_{n}^{2}}{4} \int_{-1}^{\sigma} \int_{-1}^{1} V_{\infty}\left[f^{*}(x, y+t) ; a_{i}+\eta, a_{i+1}+\eta\right]\left(1-\eta^{2}\right)^{n}\left(1-t^{2}\right)^{n} d \eta d t+ \\
& +\frac{K_{n}^{2}}{4} \int_{\sigma}^{1} \int_{-1}^{1} V_{x}\left[f^{*}(x, y+t) ; a_{i}+\eta, a_{i+1}+\eta\right]\left(1-\eta^{2}\right)^{n}\left(1-t^{2}\right)^{n} d \eta d t+ \\
& +\frac{K_{n}^{2}}{4} \int_{-\sigma}^{\sigma} \int_{\sigma}^{1} V_{x}\left[f^{*}(x, y+t) ; a_{i}+\eta, a_{i+1}+\eta\right]\left(1-\eta^{2}\right)^{n}\left(1-t^{2}\right)^{n} d \eta d t+ \\
& +\frac{K_{n}^{2}}{4} \int_{-\sigma}^{\sigma} \int_{-1}^{-\sigma} V_{x}\left[f^{*}(x, y+t) ; a_{i}+\eta, a_{i+1}+\eta\right]\left(1-\eta^{2}\right)^{n}\left(1-t^{2}\right)^{n} d \eta d t .
\end{aligned}
$$

$\left(^{20}\right)$ L. ToneliL, Cfr. nota citata in (4). 
C. VinTr: Sopra una classe di funzionali che approssimano l'area, ecc. 253

Computiamo il secondo addendo della (22):

$$
\begin{aligned}
& \frac{K_{n}^{2}}{4} \int_{-1}^{-\sigma} \int_{-1}^{1} V_{x}\left[f^{*}(x, y+t) ; a_{i}+\eta, a_{i+1}+\eta\right]\left(1-\eta^{2}\right)^{n}\left(1-t^{2}\right)^{n} d \eta d t \leq \\
₹ & \frac{K_{n}^{2}}{4}\left(1-\sigma^{2}\right)^{n} \int_{-1}^{\sigma} \int_{-1}^{1} V_{x}\left[f^{*}(x, y+t) ; a_{i}+\eta, a_{i+1}+\eta\right]\left(1-i^{2}\right)^{n} d \eta d t \leq \\
\leq & \frac{K_{n}^{2}}{4}\left(1-\sigma^{2}\right)^{n} \int_{-1}^{\sigma} \int_{-1}^{1} V_{x}\left[f^{*}(x, y+t) ; a_{i}+\eta, a_{i+1}+\eta\right] d \eta d t \leq \\
\leq & \frac{K_{n}^{2}}{4}\left(1-\sigma^{2}\right)^{n} \int_{-1}^{1} \int_{-1}^{1} V_{x}\left[f^{*}(x, y+t) ; a_{i}+\eta, a_{i+1}+\eta\right] d \eta d t= \\
= & \frac{K_{n}^{2}}{4}\left(1-\sigma^{2}\right)^{n} \int_{-1}^{1} \int_{y-1}^{y+1} V_{x}\left[f^{*}(x, \xi) ; a_{i}+\eta, a_{i+1}+\eta\right] d \eta d \xi, \text { avendo posto } y+t=\xi .
\end{aligned}
$$

Computando poi il terzo, quarto e quinto addendo della (22), si vede che anch' essi sono maggiorati dall' espressione:

$$
\frac{K_{n}{ }^{2}}{4}\left(1-\sigma^{2}\right)^{n} \int_{-1}^{1} \int_{y-1}^{y+1} V_{x}\left[f^{*}(x, \xi) ; a_{i}+\eta, a_{i+1}+\eta\right] d \eta d \xi
$$

In virtù di tali maggiorazioni, dalla (20) si deduce:

$$
\begin{gathered}
V_{x}\left[\mathscr{N} \int_{(x, y)}^{\left(\frac{1}{n}, \frac{1}{n}\right)} f^{*} ; a_{i}, a_{i+1}\right] \leq 2 M(n+1)\left(1-\sigma^{2}\right)^{n}\left(a_{i+1}-a_{i}\right)+ \\
+K_{n}^{2}\left(1-\sigma^{2}\right)^{n}+\int_{-1}^{1} \int_{y-1}^{y+1} V_{x}\left[f^{*}(x, \xi) ; a_{i}+\eta, a_{i+1}+\eta\right] d \eta d \xi+ \\
+\frac{K_{n}^{2}}{4} \int_{-\sigma}^{\sigma} \int_{-\sigma}^{\sigma} V_{x}\left[f^{*}(x, y+t) ; a_{i}+\eta, a_{i+1}+\eta\right]\left(1-\eta^{2}\right)^{n}\left(1-t^{2}\right)^{n} d \eta d t
\end{gathered}
$$


254 C. Vintr: Sopra una classe di funionali che approssinano l'area, ecc.

E poichè ̀̀ : $K_{n}^{2}=\frac{1}{\left(\int_{0}^{1}\left(1-t^{2}\right)^{n} d t\right)^{2}}<\frac{1}{\left(\int_{0}^{\sigma}\left(1-t^{2}\right)^{n} d t\right)^{2}}$, si ha :

$$
V_{x}\left[\mathscr{N}_{(x, y)}^{\left(\frac{1}{n}, \frac{1}{n}\right)} f^{*} ; a_{i}, a_{i+1}\right] \leq 2 M(n+1)\left(1-\sigma^{2}\right)^{n}\left(a_{i+1}-a_{i}\right)+
$$

$$
+K_{n}^{2}\left(1-\sigma^{2}\right)^{n}+\int_{-1}^{1} \int_{y-1}^{y+1} V_{x}\left[f^{*}(x, \xi): a_{i}+\eta, a_{i+1}+\eta\right] d \eta d \xi+
$$

$+\frac{1+\varepsilon}{4\left(\int_{0}^{\sigma}\left(1-t^{2}\right)^{n} d t\right)^{2}} \int_{-\sigma}^{\sigma} \int_{-\sigma}^{\sigma} V_{x}\left[f^{*}(x, y+t) ; a_{i}+\eta, a_{i+1}+\eta\right]\left(1-\eta^{2}\right)^{n}\left(1-t^{2}\right)^{n} d \eta_{j} d t$

essendo $\varepsilon>0$ un numero arbitrario.

Con analogo ragionamento, dalla limitazione (21), si deduce la seguente:

$$
\begin{array}{r}
V_{y}\left[\mathscr{O K}_{(x, y)}^{\left(\frac{1}{n}, \frac{1}{n}\right)} f^{*} ; c_{j}, c_{j+1}\right] \leq 2 M(n+1)\left(1-\sigma^{2}\right)^{n}\left(c_{j+1}-c_{j}\right)+ \\
+K_{n}^{2}\left(1-\sigma^{2}\right)^{n}+\int_{x+1}^{x+1} \int_{-1}^{1} V_{y}\left[f^{*}(\xi, y) ; c_{j}+t, c_{j+1}+t\right] d \xi d t+
\end{array}
$$

$+\frac{1+\varepsilon}{4\left(\int_{0}^{\sigma}\left(1-t^{2}\right)^{n} d t\right)^{2}} \int_{-\sigma}^{\sigma} \int_{-\sigma}^{\sigma} V_{\nu}\left[f^{*}(x+\eta, y) ; c_{j}+t, c_{j+1}+t\right]\left(1-\eta^{2}\right)^{n}\left(1-t^{2}\right)^{n} d \eta d t$.

Poichè ̀े $K_{n}^{2}<\frac{n+1}{\pi}\left({ }^{21}\right)$, detto $\bar{M}$ il più grande tra i numeri :

$$
\begin{aligned}
& \int_{a}^{b} d x\left\{\int_{x-1}^{x+1} \int_{-1}^{1} V_{y}\left[f^{*}(\xi, y) ; c+t, d+t\right] d \xi d t\right\} \\
& \int_{c}^{d} d y\left\{\int_{-1}^{1} \int_{y-1}^{y+1} V_{x}\left[f^{*}(x, \xi) ; a+\eta, b+\eta\right] d \eta d \xi\right\}
\end{aligned}
$$

e posto:

$$
\begin{gathered}
\varphi_{\frac{1}{n}, \frac{1}{n}}\left(y, \sigma ; a_{i}, a_{i+1}\right)=2 M(n+1)\left(1-\sigma^{2}\right)^{n}\left(a_{i+1}-a_{i}\right)+ \\
+(n+1)\left(1-\sigma^{2}\right)^{n} \int_{-1}^{1} \int_{y-1}^{y-1} V_{x}\left[f^{*}(x, \xi) ; a_{i}+\eta, a_{i+1}+\eta\right] d \eta d \xi
\end{gathered}
$$

(2i) Cfr. Ch. J. de la VAllúx-Poussin, testo citato in ${ }^{19}$ ), pag. 126. 
C. VINTI: Sopra una classe di funzionali che approssimano l'area, ecc. 255

$$
\begin{gathered}
\bar{\varphi}_{\frac{1}{n}, \frac{1}{n}}\left(x, \sigma ; c_{j}, c_{j+1}\right)=2 M(n+1)\left(1-\sigma^{2}\right)^{n}\left(c_{j+1}-c_{j}\right)+ \\
+(n+1)\left(1-\sigma^{2}\right)^{n} \int_{x-1}^{x-1} \int_{1}^{1} V_{y}\left[f^{*}(\xi, y) ; c_{j}+t, c_{j+1}+t\right] d \xi d t, \\
\psi_{h, k}(\sigma)=2 M(n+1)\left(1-\sigma^{2}\right)^{n}(b-a)(d-c)+(n+1)\left(1-\sigma^{2}\right)^{n} \bar{M},
\end{gathered}
$$

risulta:

$$
\begin{aligned}
& \sum_{i=1}^{m} \sum_{j=1}^{m^{\prime}} \int_{c_{j}}^{c_{j+1}} \varphi_{\frac{1}{n}, \frac{1}{n}}\left(y, \sigma ; a_{i}, a_{i+1}\right) d y=2 M(n+1)\left(1-\sigma^{2}\right)^{n}(b-a)(d-c)+ \\
& +(n+1)\left(1-\sigma^{2}\right)^{n} \sum_{j=1}^{m^{\prime}} \int_{c_{j}}^{c_{j+1}} d y\left\{\int_{-1}^{1} \int_{y-1}^{y+1} \sum_{i=1}^{m} V_{x}\left[f^{*}(x, \xi) ; a_{i}+\eta, a_{i+1}+\eta\right] d \eta d \xi\right\}= \\
& =2 M(n+1)\left(1-\sigma^{2}\right)^{n}(b-a)(d-c)+ \\
& +(n+1)\left(1-\sigma^{2}\right)^{n} \int_{i}^{d} d y\left\{\int_{-1}^{1} \int_{y-1}^{y+1} V_{x}\left[f^{*}(x, \xi) ; a+\eta, b+\eta\right] d \eta d \xi\right\} \leq \psi_{h, k}(\sigma), \\
& \sum_{i=1}^{m} \sum_{j=1}^{m} \int_{a_{i}}^{m_{i}} \int_{\bar{n}}^{a_{i+1}} \bar{\varphi}_{1}\left(x, \sigma ; c_{j}, c_{j+1}\right) d x=2 M(n+1)\left(1-\sigma^{2}\right)^{n}(b-a)(d-c)+ \\
& +(n+1)\left(1-\sigma^{2}\right)^{n} \sum_{i=1}^{m} \int_{a_{i}}^{a_{i+1}} d x\left\{\int_{x-1}^{x+1} \int_{-1}^{1} \sum_{j=1}^{m^{1}} V_{y}\left[f^{*}(\xi, y) ; c_{j}+t, c_{j+1}+t\right] d \xi d t\right\}= \\
& =2 M(n+1)\left(1-\sigma^{2}\right)^{n}(b-a)(d-c)+ \\
& +(n+1)\left(1-\sigma^{2}\right)^{n} \int_{a}^{b} d x\left\{\int_{x-1}^{x+1} \int_{-1}^{1} V_{y}\left[f^{*}(\xi, y) ; c+t, d+t\right] d \xi d t\right\} \leq \psi_{h, k}(\sigma) \text {. }
\end{aligned}
$$

Ricordando allora la definizione del funzionale $\overline{\mathscr{N}}\left(\sigma, \frac{1}{n}, \frac{1}{n}\right),\left(\sigma=\sigma^{*}\right.$ perchè $\left.\Theta: \sigma<1\right)$, le (23) e (24) rappresentano le (2) e (3) del N. 2. 